\title{
Pedestrian Detection in Infrared Video Images
}

\author{
Di Wu ${ }^{1,2, a^{*}}$, Wei Liu ${ }^{1, b}$ \\ ${ }^{1}$ Xi'an Institute of Optics and Precision Mechanics of CAS,Xi'an 710119,China \\ ${ }^{2}$ The University of Chinese Academy of Sciences, Beijing 100049, China \\ awudi@opt.cn, 'bliuwei@opt.ac.cn
}

\begin{abstract}
Keywords: pedestrian detection;infrared video images;background subtraction;simple features. Abstract. This paper introduces a method to detect pedestrians in infrared video images and studies its application into intelligent video surveillance. As part of a far infrared thermometric system in public places, our algorithm is aimed at the detection of human bodies in fixed area. According to the characteristics of infrared imaging technology, pedestrian detection depends on the difference of infrared radiation between the human body and the environment. Detection is achieved by the extraction and recognition of simple features of pedestrians which reduces the complexity of tradition methods and avoids using quantities of samples for training classifier. Our method not only reduces noise effectively, but also reaches high detection rate(DR) and low fault detection rate(FDR). Experimental results are presented to demonstrate the excellent performance of our algorithms.
\end{abstract}

\section{Introduction}

Pedestrian detection in images is a challenging problem in the applications of computer vision, such as intelligent video surveillance and vehicle assistance driving. Effective pedestrian detection algorithms in visible spectrum have been put forward for different systems. However, sensing in visible spectrum is infeasible under certain circumstances, meanwhile, the cost of thermal sensors has declined dramatically in the past decades. Therefore, researchers begin to focus on the area of pedestrian detection using infrared cameras and many different methods have been developed including shape-based methods [1] and motion-based methods [2].

In[3],this system exploits three detection approaches: warm area detection, edge-based detection and disparity computation. The method combines the information to create a list of relevant pedestrian candidates. Moreover, head morphological and thermal characteristics are used in searching for pedestrians.

In[4],detection is based on joint shape and appearance cues. A layered representation and a generalized EM algorithm is developed to separate infrared images into background and foreground layers. Templates with varying sizes are sequentially applied to detect pedestrians at multiple scales to accommodate different camera distances.

In[5],this method select ROIs by extracting bright regions and shape information from a whole human body. After filtering out non-pedestrian objects, the remaining ROIs are verified by a support vector machine, which uses 3554 pedestrians and 3901 non-pedestrian objects as training samples.

In[6], a real-time pedestrian detection system which relies on low quality infrared video images are presented. In order to capture the variations in human shape, probabilistic templates are introduced, especially for the case where contrast is low and body parts are missing.

In[7], a vision-based algorithm aimed at the detection of pedestrian has been presented, which is based on the localization of human shapes, size, ratio and symmetry, a distance refinement is performed using a simplified stereo-vision technique.The method is achieved on the ARGO vehicle.

In[8],the detection phase is performed by a Support Vector Machine(SVM) with size-normalized pedestrian candidates, which is further strengthened by information obtained by a road detection module that provides key information for pedestrian validation.

This paper attempts to propose an approach for pedestrian detection in infrared video images. After images denoising, ROIs is selected by background subtraction. Mathematical morphology is applied to 
eliminate the false pedestrian candidates. Later, human body recognition is achieved by simple features of human shape within ROIs.

\section{Algorithm}

Generally, Pedestrian detection algorithm consists of two stages: segmentation of ROIs and recognition of human body. The main concentration of the above papers is based on pattern recognition, using quantities of pedestrian samples to train classifier, which increases the complexity of the whole algorithm. In this paper, the region of interest(ROI) is selected by background subtraction at first, then, we apply a combination of mathematical morphology and simple features for location of pedestrian in infrared video images. The outline of our algorithm is shown in Fig.1.

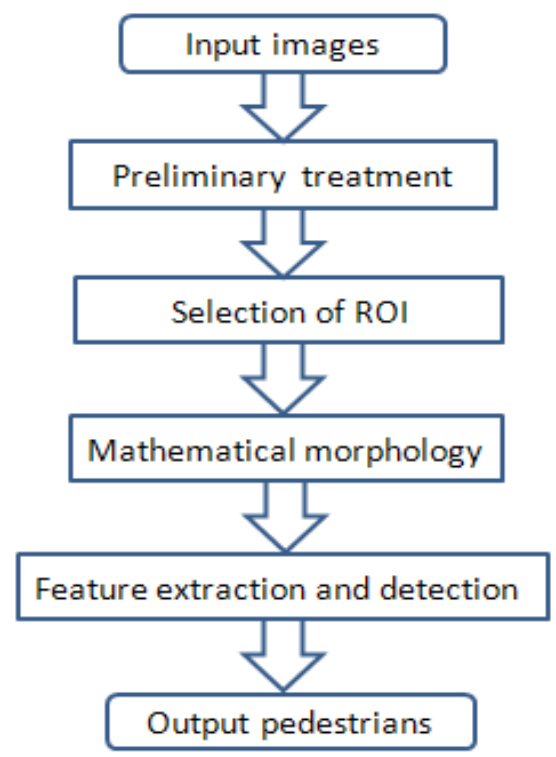

Fig.1. Outline of algorithm

Median Filtering It is necessary to preprocess the raw infrared image data, since a significant amount of noise exists in the images. Before any subsequent process, improving contrast and enhancing signal-to-noise ratio are helpful for further study. Background clutter and internal random noise impact the imaging quality of infrared imaging system. Background clutter can be simplified as Gaussian white noise, internal random noise mainly consists of thermal noise and shot noise. Median filtering can be applied to eliminate impulse noise and restore edge details, which is better for detection. In this paper, we use $3 \times 3$ median filter to cut out isolated noise.

Selection of ROIs In order to search for the exact position of pedestrians in acquired images, we need to select a potential area which may contains human bodies. The infrared image of an object depends on the amount of heat the object emits. A human body often appears as brighter districts on images due to its high temperature compared with the environment, especially for heads. Our algorithm continues with filtering for warm area through background subtraction. Background subtraction is applied to get ROIs because of stationary scene. Initially, we establish a background model by means of infrared images without moving foreground targets. Then, ROIs are obtained by subtracting the background model from the image contains pedestrians. It is calculated as Eq.1:

$$
\mathrm{g}(\mathrm{x}, \mathrm{y})=\left\{\begin{array}{l}
1, \text { if }\left|f\left(x_{i} y\right)-b(x, y)\right|>T \\
0, \quad \text { otherwise }
\end{array}\right.
$$

where $b(x, y)$ is the background model, $f(x, y)$ is the image needs to be processed, T represents the threshold. The resulting image contains only warm continuous areas which have to be verified in the next stage using mathematical morphology, since uncertainty factors exists in background modeling. 
Mathematical Morphology Due to the diversity of the pedestrian posture and motion blur of moving pedestrians, some warm patches in ROIs disturb the final decision of human bodies. Hotspots without Non-pedestrian objects are supposed to be remained for further operation. The basic operations of mathematical morphology are comprised of erosion, expansion, opening operation and closing operation. Erosion is used to cut out isolated noise which is smaller than structure element and tiny bumps on the side of contours. Expansion is used to connect tracks and fill holes in the images. Opening operation is defined as Eq.2:

$$
\mathrm{g}^{\circ} \mathrm{a}=(\mathrm{g} \odot \mathrm{a}) \oplus \mathrm{a}
$$

where $\mathrm{a}$ is the structure element applied to operate on image g obtained by ROI selection. The structure element a we use for opening operation is shown in Fig.2.

\begin{tabular}{|l|l|l|l|l|}
\hline 0 & 1 & 1 & 1 & 0 \\
\hline 1 & 1 & 1 & 1 & 1 \\
\hline 1 & 1 & 1 & 1 & 1 \\
\hline 1 & 1 & 1 & 1 & 1 \\
\hline 0 & 1 & 1 & 1 & 0 \\
\hline
\end{tabular}

Fig.2. structure element

By means of using open operation on processed binary images, we obtain candidate area with smooth edge and complete, coherent silhouette curve which has been effectively eliminated non-pedestrian objects.

Feature Detection In this section, human bodies from ROIs are verified by extracting and recognizing simple features. We consider pedestrian targets as rectangular models at this stage and segment the true target from images with two steps. Calculate the area of connected districts to eliminate the false targets, since the area of pedestrian belongs to a certain scope. Initially, we scan every pixel of the acquired binary image and mark the silhouette of each connected area, then, we utilize every boundary value to calculate the area of connected districts. The low threshold and high threshold are already known to eliminate disturbing targets out of the scope. Moreover, aspect ratio of pedestrian is applied to check which parts of connected area ought to be recognized as true targets. Generally, when the infrared camera is still, we believe the ratio of real pedestrian is from 1.4 to 5.Finally, selected pedestrians simplified as rectangular models are marked to locate the exact position of humans, contours of detected pedestrians are also presented for contrast.

\section{Experimental Results}

The proposed algorithm has been tested on an experimental platform of Intel Core $3.4 \mathrm{GHz}$ processor using Visual Studio 2010. A $288 \times 384$ uncooled far-infrared camera with sensitivity between $8 \mu \mathrm{m}$ $-14 \mu \mathrm{m}$ is used for input images. Experimental results are shown in Fig.3. As can be seen from Fig.3(a),the original image contains a lot of noise and it is obvious that the head of a human body has high values in far-infrared images. Median filtering is effective for improving SNR in Fig.3(b).ROI selection decreases the district of detection into smaller area, which is shown in Fig.3(c). After opening operation and simple features recognition, the location of pedestrian is shown in Fig.3(d).

\section{Conclusions}

A novel pedestrian detection algorithm based on infrared images is designed for intelligent video surveillance. Median filtering and background subtraction enabled ROI selection at early stages, while 
mathematical morphology was used to eliminate disturbing targets, pedestrian recognition based on simple features is implemented at last. Compared to training plenty of pedestrian samples in traditional ways, our method declines the complication and improves the efficiency dramatically.

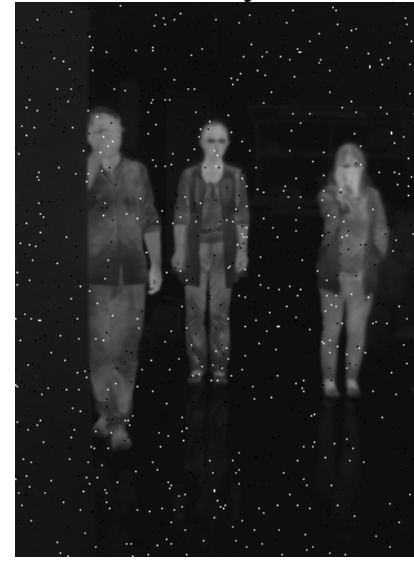

(a)

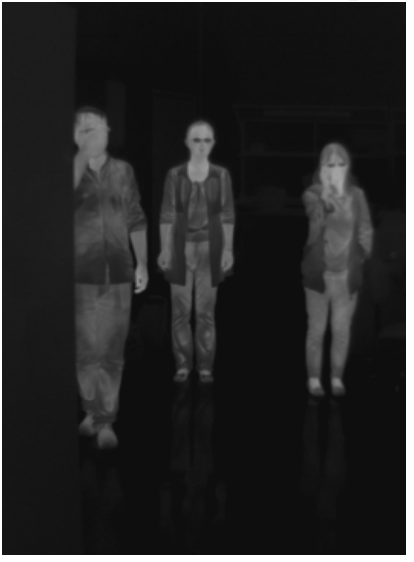

(b)

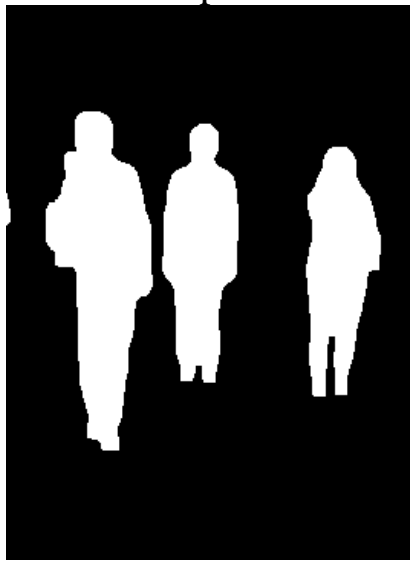

(c)

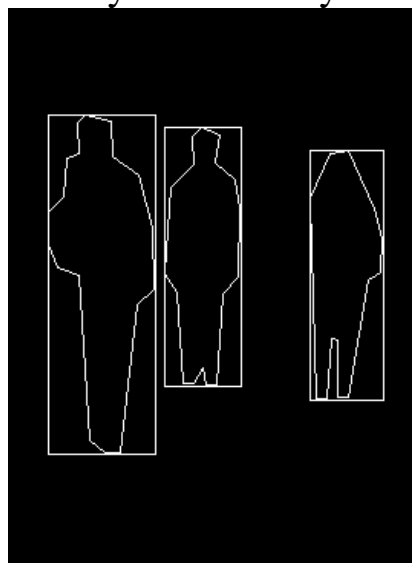

(d)

Fig.3 Detection result

This study has contributed to the successive developments of infrared thermometric system.

However, the grey level of a pedestrian depends on many factors, such as reflectivity of the clothing material and distance to the target. Fault detection rate is very high when the distance to the targets is too far or too close, for the threshold is difficult to decide. This paper only takes upright human bodies into consideration, which limits the scope of applications. In the future, we will make a combination of several different sensors to capture information from multiple domains. Moreover, hardware platforms such as DSP, FPGA will be applied to enhance the real-time of our algorithm.

\section{References}

[1] Bertozzi M, Broggi A, Caraffi C, et al. Pedestrian detection by means of far-infrared stereo vision[J]. Computer Vision \& Image Understanding, 2007, 106(23):194-204.

[2] Porikli F M, Katz J. Object segmentation using visible and infrared images: US, US7693331 B2[P]. 2010.

[3] Soundrapandiyan R, P.V.S.S.R. Chandra Mouli. Adaptive Pedestrian Detection in Infrared Images Using Background Subtraction and Local Thresholding[J]. Procedia Computer Science, 2015, 58:706-713.

[4] Dollár P, Wojek C, Schiele B, et al. Pedestrian Detection: An Evaluation of the State of the Art[J]. Pattern Analysis \& Machine Intelligence IEEE Transactions on, 2012, 34(4):743-761.

[5] Gerónimo D, López A M, Sappa A D, et al. Survey of Pedestrian Detection for Advanced Driver Assistance Systems[J]. Pattern Analysis \& Machine Intelligence IEEE Transactions on, 2010, 32(7):1239-1258.

[6] Kim D S, Lee K H. Segment-based region of interest generation for pedestrian detection in far-infrared images[J]. Infrared Physics \& Technology, 2013, 61(6):120-128.

[7] Olmeda D, Premebida C, Nunes U, et al. Pedestrian detection in far infrared images[J]. Integrated Computer-Aided Engineering, 2013, 20(4):347-360.

[8] Liu L, Bao H, Cheng X U, et al. Pedestrian Detection Method Based on MCS-LBP in Infrared Images[J]. Journal of Beijing Union University, 2015. 